\title{
Publications of Prof. Emilio Crespo
}

1977:

1. Elementos antiguos y modernos en la prosodia homérica, Salamanca, Supplement to Minos 7.

2. "La cronología relativa de la metátesis de cantidad en jónico-ático", CFC 12, 187219.

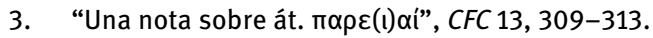

4. Review of J.L. Perpillou, Les substantifs grecs en -عúৎ (Paris, 1973), in: Minos 16, 257-249.

5. Review of J.L. García Ramón, Les origines postmycéniennes du groupe dialectal éolien. Étude linguistique (Salamanca, 1975), CFC 13, 319-321.

6. Review of C.E. Hajistephanou, The use of $\varphi$ úoı and its cognates in Greek tragedy with special reference to character drawing (Nicosia, 1975), CFC 13, 322-323.

1979:

7. Heliodoro, Las etiópicas o Teágenes y Cariclea, translation, foreword and notes, Madrid, Gredos. Reprint without introduction, Barcelona, Planeta-Agostini, and partial reprints in: Antología de la literatura griega, selection and introduction by Carlos García Gual and Antonio Guzmán Guerra, Madrid, 1995, Alianza Editorial. Reprint with new foreword, Madrid, 2002, Biblioteca Básica Gredos.

8. “La alternancia $\Sigma \Sigma / T T$ y la prosa literaria ática del siglo V a. C.”, CFC 16, 109-125.

1980:

9. Review of S.T. Teodorsson, The phonology of Attic in the Hellenistic Period (Göteborg Uppsala, 1975), Emerita 48, 145-148.

1981:

10. “Sobre el orden de palabras en griego: el genitivo adnominal”, Emerita 49, 33-65.

1982:

11. Jámblico, Babiloníacas. (Fotius’ Abstract and Fragments), Madrid.

1983:

12. Plutarco, Vidas paralelas (Alejandro-César, Pericles-Fabio Máximo, Alcibíades Coriolano), translation, foreword and notes, Barcelona, 1983, Bruguera. Published with additions and corrections, Madrid, 1999, Cátedra. Partial reprints in: Antología de la literatura griega, selection and introduction by Carlos García Gual.

13. “Una clasificación de los adjetivos en griego", Emerita 51, 301-313.

14. "Sobre el orden de palabras en griego: de SOV hacia SVO", Actas del VI Congreso Español de Estudios Clásicos II, Madrid, 287-294.

15. Review of $\mathrm{L}$. Basset, Les emplois périphrastiques du verbe grec $\mu \varepsilon \dot{\lambda} \lambda \varepsilon \imath \mathrm{v}$. Étude de linguistique grecque et essai de linguistique générale (Lyon, 1979), Emerita 51, 353355. 
1984:

16. "Infinitivo modal sin ớv en griego", in: Rosa M. Aguilar/Luis Gil (eds), Apophoreta philologica M. Fernández-Galiano a sodalibus oblata I (EClas 87), Madrid, 67-73.

17. "Sintaxis griega", in: Alfonso Martínez (ed.), Actualización científica en Filología griega, Madrid, 321-353.

18. "Sobre las Vidas de Filopemen y Flaminino de Plutarco", in: Manuel Fernández-Galiano (ed.), Auguralia. Estudios de lenguas y literaturas griega y latina, Madrid, 6172.

19. "On the system of the substantive clauses in Ancient Greek: A functional approach", Glotta 62, 1-16.

20. “La conjunción ö $\varphi \rho \alpha$ : significado y contexto", in: Luis A. de Cuenca et al. (eds), Athlon. Satura grammatica in honorem Francisci R. Adrados, vol. I, Madrid, 129-138.

1985:

21. “Palatal Stops in Greek: Reconstruction or Mycenaean Evidence?”, Minos 19, 91104.

22. Review of J.O. Rosenqvist, Studien zur Syntax und Bermekungen zum Text der Vita Theodori Syceotae (Uppsala, 1981), Emerita 53, 175-176.

23. Review of A. Rijksbaron, The Syntax and Semantics of the Verb in Classical Greek. An Introduction (Amsterdam, 1984), Kratylos 30, 188-190.

1986:

24. "Aristóteles, Física II 1 (192 b 8 - 193 b 21) (ed. Ross): traducción y comentario", (Crespo et al.), Sociedad española de profesores de Filosofía de Instituto. Boletín informativo 19, 11-18.

25. Review of M. Brioso Sánchez (ed.), Bucólicos griegos (intr., trad. y notas por M. García Teijeiro y M.T. Molinos Tejada) (Madrid, 1986), in: Delegación de Madrid de la SEEC. Boletín informativo 16, 323.

1987:

26. Review of E. Garrido González (ed.), La mujer en el mundo antiguo. Actas de las Quintas Jornadas de Investigación Interdisciplinaria (Madrid, 1986), in: Delegación de Madrid de la SEEC. Boletín informativo 17, 26-26.

27. Review of F.M.J. Waanders, The History of TELOS and TELES in Ancient Greek (Amsterdam, 1983), Emerita 55, 159-161.

28. Review of J.S. Clay, The Wrath of Athena. Gods and Men in the Odyssey (Princeton, 1983), Emerita 55, 363-365.

1988:

29. Alcibíades. Antología de textos con notas y comentarios, edition of E. Crespo et al, Madrid.

30. "Selección de textos de la Antigüedad sobre las Olimpíadas en Grecia”, Historia 16, $106,1-8$.

31. “La expresión de la finalidad en las subordinadas del griego antiguo", REL 18, 285298. 
32. "The Semantic and Syntactic Functions of the Accusative", in: Albert Rijksbaron et al. (eds), In the Footsteps of Raphael Kühner. Proceedings of the International Colloquium in Commemoration of the 150th Anniversary of the Publication of Raphael Kühner's Ausführliche Grammatik der griechischen Sprache. II: Syntaxe, Amsterdam, 99-120.

33. “La expresión de la función Manera en griego”, Emerita 56, 43-64.

34. 'La 'Carta de Claudio a los alejandrinos' (P. Lond. 1912): un tipo de koiné en el Egipto de época de Claudio", Minerva 2, 213-231.

35. Review of M.H.B. Marshall, Verbs, Nouns, and Postpositives in Attic Prose (Edinburgh, 1987), Emerita 56, 337-339.

1989:

36. “Mesa redonda sobre Didáctica del griego. Presentación”, Actas del VII Congreso Español de Estudios Clásicos III, Madrid, 709-717.

37. "Participio absoluto y subordinada adverbial”, Actas del VII Congreso Español de Estudios Clásicos I, Madrid, 119-126.

1990:

38. “Ante la reforma de las enseñanzas universitarias de Filología Clásica”, in: Francisco Rodríguez Adrados, (ed.), Didáctica de las Humanidades Clásicas ante la Reforma de la Enseñanza, Madrid, 5-14.

39. "El final de la guerra del Peloponeso: notas históricas a Plutarco, Vida de Lisandro, 13-18", in: Aurelio Pérez Jiménez/Gonzalo del Cerro (eds), Estudios sobre Plutarco: obra y tradición. Actas del I Symposion español sobre Plutarco, Málaga, 63-71.

40. Review of M.S. Ruipérez y J.L. Melena, Los griegos micénicos (Madrid, 1990), Historia 16 15, n. 174.

1991:

41. Homero, Iliada, translation, foreword and notes, Madrid, 1991, Gredos. Reprints without the foreword in Barcelona, 1995, Círculo de Lectores; and Barcelona, 1997, Planeta-Agostini. With a new short foreword, Madrid, 2000, Biblioteca Básica Gredos. With the Greek text of the Iliad and of the Odyssey and with the translation by J.M. Pabón of the Odyssey and foreword by C. García Gual, Madrid, 1999, Espasa Calpe. Partial reprints in: Antología de la literature griega, selection and introduction by C. García Gual and A. Guzmán Guerra, Madrid, 1995, Alianza Editorial. Many reprints in Gredos and later in RBA Publishing Houses.

1992:

42. Homerica. Estudios lingüísticos, edition in collaboration with José Luis García Ramón/Helena Maquieira/Jesús de la Villa, Madrid.

43. “Sintaxis y semántica de las formas modales en griego clásico”, REL 22, 277-307.

44. "El uso de los temas de aoristo y de presente para la expresión de la repetición distributiva”, in: Emilio Crespo et al. (eds), Homerica. Estudios lingüísticos, Madrid, 1334.

45. Review of L. Basset, La syntaxe de l'imaginaire. Étude des modes et des negations dans l'lliade et l'Odyssée (Lyon, 1989), Emerita 60, 162-164. 
46. Review of M. Casevitz, Études homériques (Lyon/Paris, 1989), Emerita 60, 1992, 344-346.

47. Review of J.L. García Ramón (ed.), M. S. Ruipérez, Opuscula selecta. Ausgewählte Arbeiten zur griechischen und indogermanischen Sprachwissenschaft (Innsbruck, 1989), EClas 34, 182-184.

1993:

48. Dialectologica Graeca. Actas del II Coloquio internacional de dialectología griega, edition in collaboration with José Luis García Ramón and Araceli Striano, Madrid.

49. "Two Epigraphic Uses of the Verbal Moods in the Embedded Predications", in: Emilio Crespo/José Luis García Ramón/Araceli Striano (eds), Dialectologica Graeca. Actas del II Coloquio internacional de dialectología griega, Madrid, 91-109.

50. Review of J. Latacz (ed.), Zweihundert Jahre Homer-Forschung. Rückblick und Ausblick, Colloquium Rauricum, Band 2 (Stuttgart/Leipzig, 1991), Emerita 61, 385 ff.

1994:

51. "Datos para un estudio contrastivo del aspecto gramatical en griego clásico y en moderno", in: Luis M. Macía et al. (eds), Quid ultra faciam? Trabajos de griego, latín e indoeuropeo en conmemoración de los 25 años de la UAM, Madrid, 33-37.

52. “L'expression de l'accompagnement en grec ancien”, in: Bernard Jacquinod (ed.), Cas et prépositions en grec ancien: contraintes syntaxiques et interprétations sémantiques. Actes du Colloque international de Saint-Etienne, 3-5 juin 1993, SaintÉtienne, 181-192.

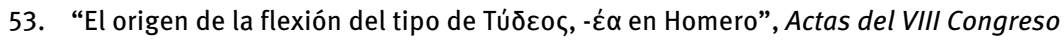
Español de Estudios Clásicos I, Madrid, 87-92.

54. “Plutarco y la epigrafía”, in: Rosa M. Aguilar/Mercedes López Salvá/Ignacio

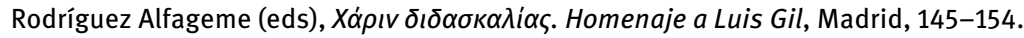

55. Review of A. López Eire, Ático, koiné, y aticismo. Estudios sobre Aristófanes y Libanio (Murcia, 1991), Emerita 62, $197 \mathrm{ff}$.

1995:

56. “Actualidad de las lenguas clásicas”, Vela Mayor 6, 11-18.

57. "Bibliografía sobre sintaxis griega 1985-1994", Tempus 10, 5-18.

58. “Bibliographie sur la syntaxe grecque 1985-1994", Syntaktika 9, 1-14.

59. "Respuestas al cuestionario sobre el caso dativo", in: Esperanza Torrego et al. (eds), Sintaxis del dativo latino. I Encuentro de sintaxis latina (10-11 de junio de 1994), Madrid/Barcelona, 107-110.

60. Review of P. Hummel, La syntaxe de Pindare (Louvain/Paris, 1993), Emerita 63, 363 ff. 
1996:

61. “Introducción a la lectura de Las etiópicas de Heliodoro", Nova Tellus 14, 129-152.

62. "Los problemas de la sintaxis del griego antiguo", in: Ana Agud/José Antonio Fernández Delgado/Agustín Ramos (eds), Las lenguas de corpus y sus problemas lingüísticos, Madrid, 167-175.

63. “Textos sobre el paisaje de Grecia en la Antigüedad”, EClas 110, 33-56.

1997:

64. Berthold Delbrück y la sintaxis indoeuropea hoy. Actas del coloquio de la Indogermanische Gesellschaft, edition in collaboration with José Luis García Ramón, Madrid/Wiesbaden.

65. “Delbrück y la sintaxis de los modos”, in: Emilio Crespo/José Luis García Ramón (eds), Berthold Delbrück y la sintaxis indoeuropea hoy. Actas del Coloquio de la Indogermanische Gesellschaft, Madrid, 21-24 de septiembre de 1994, Madrid/Wiesbaden, 27-61.

66. “L’ordre de préférence des éléments linguistiques de l'épopée”, in: François Létoublon/Helma Dik (eds), Hommage à Milman Parry. Le style formulaire de l'épopée homérique et la théorie de l'oralité poétique, Amsterdam, 129-135.

67. "Sintaxis de los elementos de relación en griego clásico", in: Francisco Rodríguez Adrados (ed.), Actas del IX Congreso Español de Estudios Clásicos, Madrid, 3-42.

68. Review of P. Cuzzolin, Sull'origine della costruzione dicere quod: aspetti sintattici e semantici (Firenze, 1994), Emerita 65, 339-341.

69. Review of E. Redondo Moyano, Estudio sintáctico de las partículas en el período helenística: Herodas, EClas 39, n. 112, 179-181.

1998 :

70. Los dioses del Olimpo, edition in collaboration with Grupo Tempe, Madrid, $2^{\mathrm{a}}$ ed. 2016.

71. “La construcción del Partenón”, in: Crescente López de Juan/Domingo Plácido (eds), Momentos estelares del Mundo Antiguo, Madrid, 61-79.

72. “Los sintagmas nominales en aposición oracional y la descripción semántica de la oración”, in: Luis Gil/Marcos Martínez (eds), Corolla Complutensis. Homenaje al profesor J.S. Lasso de la Vega, Madrid, 63-66.

73. "Niveles sintácticos de la estructura de la oración principal simple en griego clásico", in: Esperanza Torrego (ed.), Nombres y funciones: estudios de sintaxis griega y latina, Madrid, 43-63.

74. Review of P.A. Mumm, Parameter des einfachen Satzes aus funktionaler Sicht. Abriß ihrer onomasiologischen Systematik. Teil I: Relationierung der Lexeme in der Prädikation; Valenz, Numeralität und Aspektualität des Verbs (München/Newcastle, 1996), Kratylos 43, 47-53. 
1999:

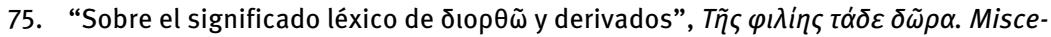
lánea léxica en memoria de Conchita Serrano, Madrid, 61-65.

76. “Paramètres pour la définition des complétives en grec ancien”, in: Bernard Jacquinod (ed.), Les complétives en grec ancien, Saint-Étienne, 45-62.

77. “Cronología de los segundos alargamientos compensatorios en jónico-ático", in: Albio C. Cassio (ed.), Katà diálekton. Atti del III Colloquio Internazionale di dialettologia greca, Naples, 61-186.

78. “llíada, 20.419-454: el duelo fallido entre Aquiles y Héctor”, in: Juan Antonio López Férez (ed.), Desde los poemas homéricos hasta la prosa griega del siglo IV d.C. Veintiséis estudios filológicos, Madrid, 1-10.

2000:

79. Actas del X Congreso Español de Estudios Clásicos, volumen I, edition in collaboration with $M^{\mathrm{a}}$ José Barrios Castro, Madrid.

80. "La lengua y la escritura en las utopías de la literatura griega antigua”, in: Minerva Alganza (ed.), EПIEIKEIA: Homenaje al profesor Jesús Lens Tuero, Granada, 89-94.

81. “Panorama de la retórica y de la poética griegas en época clásica”, in: F. Sevilla (ed.), Edad de Oro XIX, 65-81.

82. "Funciones categoriales", in: Marcos Martínez et al. (eds), Cien años de investigación semántica: de Michel Bréal a la actualidad. Actas del congreso internacional de semántica, Madrid, 1291-1299.

83. Review of C.M.J. Sicking/P. Stork, Two Studies in the Semantics of the Verb in Classical Greek (Leiden/New York/Köln, 1996), Kratylos 45, 122-127.

2001

84. Review of Ilja Leonard Pfeijffer, First Person Futures in Pindar (Stuttgart, 1999), Emerita 69, $179 \mathrm{ff}$.

2002:

85. "Los mitos en la Ilíada", in: Juan Antonio López Férez (ed.), Mitos en la literatura griega arcaica y clásica, Madrid, 35-54.

2003:

86. Sintaxis del griego clásico, in collaboration with Luz Conti and Helena Maquieira, Madrid.

87. "El significado de $̋ \sigma t \varepsilon$ o $\dot{\omega} \varsigma$ con verbo en infinitivo o en forma personal”, in: Jesús María Nieto (ed.), Lógos Hellenikós. Homenaje al profesor Gaspar Morocho Gayo I, León, 97-104.

88. "Los eolismos en la lengua homérica", Nova Tellus 21, 15-46.

89. "La sintaxis griega hoy", Synthesis 10, 31-53.

90. Review of B. Jacquinod et al., Études sur l'aspect chez Platon (Saint-Étienne, 2000), Kratylos 48, 118-122.

91. Review of André Sauge, Les degrés du verbe. Sens et formation du parfait en grec ancien (Bern/Berlin/Bruxelles/Frankfurt am Main/New York/Oxford/Wien, 2000), Kratylos 48, 206-209. 
92. Review of Aristóteles, Poética. Introducción, traducción, notas y comentario de A. López Eire. Epílogo de J.J, Murphy (Madrid, 2002), Estudios Clásicos 124, 112-114.

2004:

93. Obras completas de Esquilo, Sófocles y Eurípides, edition with foreword and notes, in collaboration with Luz Conti, Rosario López, Luis M. Macía and Ma Eugenia Rodríguez, Madrid.

94. "Los nombres de los troyanos y de los griegos en la llíada", Classica, Belo Horizonte $17-18,33-47$.

95. "The Attitude of the Athenian State towards the Attic Dialect in the Classical Era", in: J.H.W. Penney (ed.), Indoeuropean Perspectives. Studies in Honour of Anna Morpurgo Davies, Oxford, 109-118.

96. "La lengua como instrumento político en la Atenas clásica”, in: Ana María González de Tobía (ed.), Ética y estética: de Grecia a la modernidad, La Plata, 41-58.

97. Review of P. Cabrera/R. Olmos (coords.), Sobre la Odisea. Visiones desde el mito y la arqueología (Madrid, 2003), Estudios Clásicos 126, 112-114.

2005:

98. "Política lingüística en la Antiguedad clásica”, in: Antonio Alvar/José Francisco González Castro (eds), Actas del XI Congreso de la Sociedad Española de Estudios Clásicos I, Madrid, 87-109.

99. “La actualidad de la llíada: de la poesía oral a internet”, Letras Clássicas (Sao Paulo) $5,29-61$.

100. Review of G.L. Cooper, Attic Greek Prose Syntax (vol. 1-2) and Greek Syntax: Early Greek Poetic and Herodotean Syntax (volumes 3-4) (Ann Arbor 1998 and 2003), Ordia Prima 4, 177-179.

2006:

101. Word Classes and Related Topics in: Ancient Greek, edition in collaboration with Jesús de la Villa and Antonio Revuelta, Louvain-la-Neuve.

102. "El Partenón a través de los textos y de las imágenes", in: J.E. Rojas (ed.), III Jornadas filológicas. Homenaje a Noel Olaya Perdomo, Bogotá, 43-69.

103. "The Language Policy of the Athenian State in the Fifth Century B.C.", Incontri Linguistici 29, 91-101.

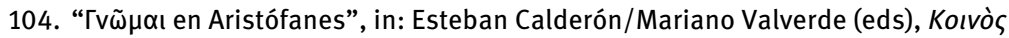
ヘóyoc. Homenaje al profesor José García López, Murcia, 197-201.

105. "Clases de palabras en griego antiguo: estado de la cuestión y algunos problemas pendientes”, in: Emilio Crespo/Jesús de la Villa/Antonio Revuelta (eds), Word Classes and Related Topics in Ancient Greek. Proceedings of the Conference on 'Greek Syntax and Word Classes' held in Madrid on 18-21 June 2003, Louvain-la-Neuve, 925.

106. "La cólera de Aquiles", biTARTE 38, 61-75.

107. Review of R.J. Allan, The Middle Voice in Ancient Greek. A Study in Polysemy (Amsterdam, 2003), Gnomon 78, 683-868.

108. Review of S. Segura Munguía, Los jardines en la Antigüedad (Bilbao, 2005), in: Iris. 
109. Review of L.M. Pino Campos, Estudios sobre María Zambrano: el magisterio de Ortega y las raíces grecolatinas de su filosofía (La Laguna, 2005), Revista de hispanismo filosófico 11, 196-198.

2007:

110. El Banquete, de Platón, Madrid.

111. “The Linguistic Policy of the Ptolemaic Kingdom”, in: Miltiades B. Hatzopoulos (ed.),

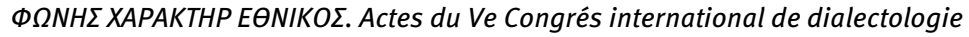
grecque (Athènes, 28-30 septembre 2006), Athens, MENETHMATA 52, 35-49.

112. "La epopeya nacional en la literatura clásica y en el cine: La bataglia di Algeri de Gillo Pontecorvo", in: Carmen González/Luis Unceta (eds), Literatura clásica, estética y cine contemporáneo: épica, Madrid, 107-112.

113. Review of A. Willi, The Languages of Aristophanes. Aspects of Linguistic Variation in Classical Attic Greek (Oxford, 2003), Kratylos 52, 70-75.

114. Review of J. Luque Moreno, Puntos y comas: la grafía de la articulación del habla (Granada, 2006), The Classical Bulletin 83, 337-338.

\section{8:}

115. "Léxico y temas en las Suplicantes de Esquilo", in: J. Vicente Bañuls/Francesco de Martino/Carmen Morenilla (eds), Teatro y sociedad en la Antigüedad clásica. Las relaciones de poder en época de crisis, Bari, 119-127.

116. "Latín obligatorio», in: Antonio Cascón et al. (ed.), Donum amicitiae. Estudios en homenaje al profesor Vicente Picón García, Madrid, 109-116.

117. “Language Policy in Classical Athens”, in: Ilze Rumniece (ed.), Hellēṇu Mantojums (Hellenic Heritage), Riga, 19-27.

118. "Política lingüística en la antigüedad clásica: el reino de los Ptolomeos de Egipto", in: Omar Daniel Álvarez Salas (ed.), Cultura clásica y su tradición. Balance y perspectivas actuales I, México, 83-100.

119. “El Partenón a través de textos e imágenes”, in: Guadalupe Fernández Ariza (ed.), Literatura hispanoamericana del siglo XX. Literatura y arte, Málaga, 9-35.

120. "L’adverbe हैıı dans les dialectes grecs", in: René Hodot/Guy Vottéro (eds), Dialectes grecs et aspect verbal. Actes de la table ronde de Saint-Etienne, 17-18 juin 2004, Nancy, 29-38.

2009:

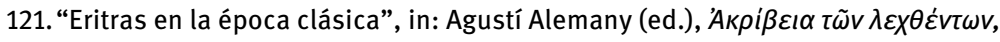
Homenatge a Rosa-Araceli Santiago, Faventia 31.1-2, 171-177.

122. "La difusión temprana del dialecto ático en el Peloponeso", in: Ángel Martínez Fernández (ed.), Estudios de epigrafía griega, La Laguna, 137-143.

123. “Conjunctive Adverbs in: Ancient Greek”, in: Katerina Loudová/Marie Žáková (eds), Early European Languages in the Eyes of Modern Linguistics, Brno, 111-120.

124. "La traducción de la literatura grecolatina al español hoy", in: Veronika Zondek/ Amalia Ortiz (eds), Escrituras de la traducción hispánica, Valdivia, 167-174.

125. "Prólogo a esta edición”, in: Albin Lesky, Historia de la literatura griega, I. De los comienzos a la polis griega, Madrid, 9-17. 
126. "Tema de presente", "Tema de Aoristo" and "Tema de futuro" in: Morfología Griega; “Introducción”, in: Sintaxis Griega Madrid, Liceus <http://www.liceus.com/cgi-bin/ aco/culc/tema_2.asp\#linguistica>.

127. “Homero", in: Francisco Lafarga (ed.), Diccionario de la historia de la traducción en España, Madrid.

128. Review of J. Pòrtulas, Introducció a la llíada. Homer, entre la història i la llegenda (Barcelona, 2008), Estudios Clásicos 135, 117-118.

129. Review of I. Rodríguez Alfageme, Aristófanes: escena y comedia (Madrid, 2008), in: Iris.

130. Review of J.M. Baños Baños (coord.), Sintaxis del latín clásico (Madrid, 2009), Myrtia 24, 409-415.

2010:

131. "El proceso de configuración y fijación de la koiné en el siglo IV", in: Francisco Cortés/Julián V. Méndez Dosuna (eds), Dic Mihi, Musa, Virum. Homenaje al profesor Antonio López Eire, Salamanca, 139-146.

132. "The Significance of Attic for the Continued Evolution of Greek", in: Chrys Caragounis (ed.), Greek, A Language in Evolution. Essays in Honour of Antonios N. Jannaris, Hildesheim/Zurich/New York, 119-136.

133. Cultura Clásica (www.wikisaber.es).

134. Review of L. Gil Fernández, Sobre la democracia ateniense (Madrid, 2009), Minerva $23,285-287$.

2011:

135. "Pour une définition des propositions relatives latines", LEC 79, 21-34.

136. "OF fr. 496: dialectal diversity in Macedon at the end of the fourth century B.C.", in: Miguel Herrero et al. (eds), Tracing Orpheus: Studies of Orphic Fragments in Honour of Alberto Bernabé, Berlin/Boston, 227-230.

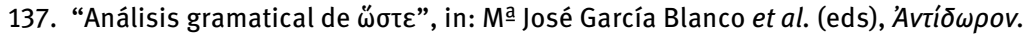
Homenaje a Juan José Moralejo, Santiago de Compostela, Universidade de Santiago de Compostela, 141-152.

138. “Conjunctive Adverbs: A Neglected Chapter of Greek Grammar”, in: Eugenio Luján/ Juan Luis García Alonso (eds), A Greek Man in the Iberian Street. Papers in Linguistics and Epigraphy in Honour of Javier de Hoz, Innsbruck, 35-43.

139. "Viajando por obligación: las heroínas en la novela griega”, in: Rosario López Gregoris/Luis Unceta (eds), Ideas de mujer. Facetas de los femenino en la Antigüedad, Alicante, 159-170.

140. "La filiación en Homero y en Hesiodo", in: Patricio de Navascués et al. (eds), Filiación. Cultura pagana, religión de Israel, orígenes del cristianismo III (Actas de las V y VI Jornadas de estudio "La filiación en los inicios de la reflexión cristiana, Instituto de Filología San Justino"), Madrid, 37-47.

141. "Sobre Lisias XVI (Defensa de Mantíteo)", in: Silvia Aquino et al. (eds), La fascinación por la palabra. Homenaje a Paola Vianello, México, 125-134.

142. Short biographies of Martín Ruipérez and Antonio Ruiz de Elvira, in: Diccionario biográfico español, Real Academia de la Historia. 
2012:

143. Platón, Menéxeno. Discursos en honor de los caídos por Atenas, bilingual edition, introduction and notes by Alberto Enrique Álvarez, Raquel Fornieles, María González, Mireia Movellán and Juan Muñoz. Under the direction of Emilio Crespo, Madrid, Dykinson, 2012.

144. "Las traducciones de Homero en América Latina. As traduções de Homero na América Latina", in collaboration with J. Piqué, in: Helena Maquieira/Claudia Fernández (eds), Tradición y traducción clásica en América Latina, La Plata, 237-242.

145. "Languages and Dialects in Ancient Macedon", in: Georgios K. Giannakis (ed.), An-

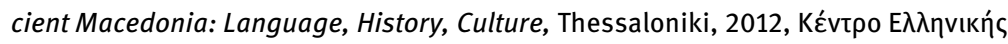

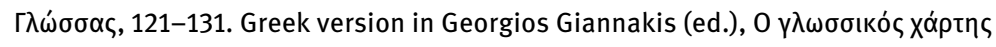

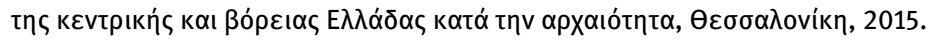

146. Review of A. Blanc, Les contraintes métriques dans la poésie homérique. L'emploi des thèmes nominaux sigmatiques dans l'hexamètre dactylique (Paris 2008), in CFC (eg) 22, $240 \mathrm{ff}$.

2013:

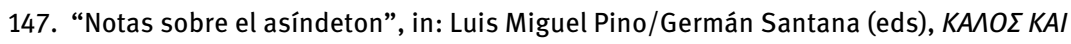

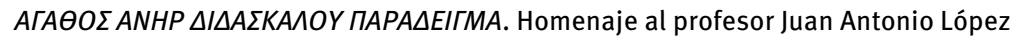
Férez, Madrid, 213-216.

148. "Una nueva llíada". Review of F. Javier Pérez, Homero, Ilíada (Madrid, 2012), Revista de libros.

2014:

149. Encyclopedia of Ancient Greek Language and Linguistics, edition in collaboration with Georgios K. Giannakis (General Editor)/V. Bubenik/Ch. Golston/A. Lianeri/ S. Luraghi/S. Matthaios (Associate Editors), Leiden.

150. "Semblanzas: Manuel García Teijeiro: lingüística" and "De adverbio a conjunción coordinante", in: Ángel Martínez Fernández et al. (eds), AGALMA. Ofrenda desde la Filología Clásica a Manuel García Teijeiro, Valladolid, 2014, 52-53 y 135-141.

151. "Ordinal Adverbs as Markers of Discourse Cohesion”, in: Alfred Bamesberger/ 0 . Hackstein/Sabine Ziegler (eds), Von Fall zu Fall. Beiträge zur indogermanischen Syntax, Wiesbaden, 81-89.

152. "Diffusion de l' attique et développement de koinai dans le Péloponnèse ( $1^{\text {re }}$ moitié du IV siècle av. J.-C.)", in: Sophie Minon (ed.), Diffusion de l'attique et expansion des koinai dans le Péloponnèse et en Grèce centrale. Actes de la journée internationale de dialectologie grecque du 18 mars 2011, Geneva, 57-68.

153. "A Rule for the Choice of Aorist and Imperfect", in: Annamaria Bartolotta (ed.), The Greek Verb. Morphology, Syntax, and Semantics. Proceedings of the $8^{\text {th }}$ International Meeting on Greek Linguistics, Agrigento, October 1-3, 2009, Louvain-la-Neuve, 7182. 
154. "Atenea como personificación del Estado ateniense en las estelas áticas inscritas",

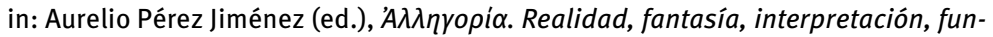
ciones y pervivencia del mito griego. Estudios en honor del Profesor Carlos García Gual, Zaragoza, 15-122.

155. "La copa de Néstor y la datación de la llíada", in: Pedro Bádenas et al. (eds), Per speculum in aenigmate. Miradas sobre la Antigüedad. Homenaje a Ricardo Olmos, Madrid, Anejos de Erytheia. Estudios y textos 7, 73-78.

2015:

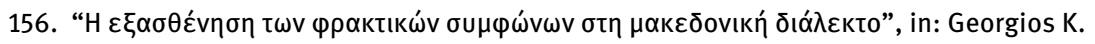

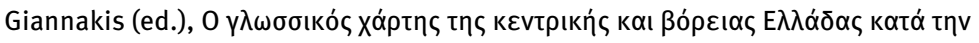

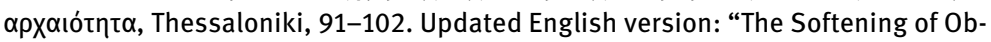
struent Consonants in Macedonian”, in: Georgios K. Giannakis/Emilio Crespo/ Panagiotis Filos (eds), Studies in Ancient Greek Dialects: From Central Greece to the Black Sea, Berlin/New York, 2018, 329-348.

157. “In memoriam Martín Ruipérez Sánchez”, EClas 148, 95-101.

158. "Foreword" to Jorge Bergua Cavero, Pronunciación y prosodia del griego antiguo. Guía práctica para la lectura de sus textos, Madrid, Ediciones Clásicas.

159. "El teatro de Dioniso y la política ateniense", in: Antonio Alvar (ed.), La vida a escena: Ayer y hoy del teatro clásico, Madrid, 27-32.

160. "Foco informativo y foco contrastivo en griego clásico", in: Jesús Ángel y Espinós et

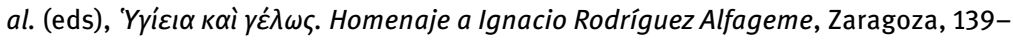
150.

161. "Introduction" to the academic session on "Greek and Latin Linguistics", Minerva 28, 13-15.

162. "La aparición del concepto de cuerpo en época posthomérica”, in: Carmen Sánchez/ Inmaculada Escobar (eds), Dioses, héroes y atletas. La imagen del cuerpo en la Grecia antigua, Madrid, 45-51.

163. "Adverbios de foco en griego clásico", in: José Vela/Juan Francisco Fraile/Carmen Sánchez (eds), Studia Classica Caesaraugustana: Vigencia y presencia del mundo clásico hoy: XXV años de Estudios Clásicos en la Universidad de Zaragoza, Zaragoza, 207-233.

164. "Los adverbios conjuntivos en griego", in: Jesús de la Villa et al. (eds), lanua Classicorum: temas y formas del mundo clásico, Madrid, 2015, 485-494.

165. “Semblanza”, in: Gerardo Ramírez/Aurelia Vargas (eds), La ritualidad de la palabra, México, 13-20.

2016:

166. "Los exiliados de la guerra civil española en Latinoamérica y los estudios clásicos", Revista de la Universidad de La Habana 282, 10-20.

167. "Languages in Ancient Egypt”, in: Sofía Torallas/Alberto Nodar (eds), Pharaoh's Reeds. A Papyrus Voyage up the Nile, Barcelona 2016, 34-40.

168. "Sobre el teatro ateniense y la configuración de la ideología cívica”, EYПOIKIAON ANOO乏. Estudios sobre teatro griego en homenaje a Antonio Melero, Studia Philologica Valentina $18.15,51-56$. 
169. "La construcción de dos o más sintagmas nominales coordinados en nominativo con verbo en singular", in: Elena Redondo/Ma José García Soler (eds), Nuevas interpretaciones del mundo antiguo. Papers in Honor of Professor José Luis Melena on the Occasion of his Retirement, Anejos de Veleia, Series Minor 33, Vitoria, 101-110.

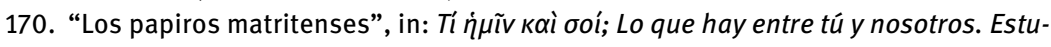
dios en honor de María Victoria Spottorno, Digitalia Antiqua 1 Córdoba, 87-94.

171. "Tres epigramas griegos", Anáfora 9, 17-20.

2017:

172. La idea de lo clásico, edition in collaboration with Pedro Aullón de Haro, Madrid, Casimiro.

173. "A Unitary Account of the Meaning of kai", in: Camille Denizot/Olga Spevak (eds), Pragmatic Approaches to Latin and Ancient Greek, Amsterdam-Philadelphia, 257272.

174. “Focus Adverbs in Classical Greek”, in: Felicia Logozzo/Paolo Poccetti (eds), Ancient Greek Linguistics: New Approaches, Insights and Perspectives, Amsterdam/Philadelphia, 133-154.

175. “Le future dorien dans l'épopée archaïque”, in: Rutger J. Allan/Frédéric Lambert/ Theodore Markopoulos (eds), The Greek Future and its History, Louvain-la-Neuve, 35-41.

176. "Adverbios como modificadores del sintagma nominal en griego clásico", in: Germán Santana/Luis Miguel Pino, חAISEIA KAI ZHTHEIS. Homenaje a Marcos Martínez, Madrid, 199-204.

177. "Los orígenes de la idea de lo clásico en la cultura occidental”, in: Pedro Aullón de Haro/Emilio Crespo (eds), La idea de lo clásico, Madrid, 37-48.

178. «La historicidad de la guerra de Troya: progresos recientes", in: Juan Piquero/Jesús Quílez (eds), Desmontando mitos ¿Ocurrió realmente como nos lo han contado?, Madrid, 13-28.

179. "Prefacio", in: Ma Jesús Albarrán/Raquel Martín/Irene Pajón (eds), Estudios papirológicos: textos literarios y documentales del siglo IV a.C. al siglo IV d. C., Madrid.

2018:

180. Phílos hetaîros. Homenaje al profesor Luis M. Macía, edition in collaboration with Luz Conti, Ma Eugenia Rodríguez, Esperanza Torrego and Jesús de la Villa, Madrid.

181. Studies in Ancient Greek Dialects: From Central Greece to the Black Sea, edition in collaboration with Georgios K. Giannakis and P. Filos, Berlin/Boston.

182. "Los enunciados parentéticos en la llíada”, in Luz Conti/Emilio Crespo/MMa Eugenia Rodríguez/Esperanza Torrego/Jesús de la Villa (eds), Phílos hetaîros. Homenaje al profesor Luis M. Macía, Madrid, 219-228.

183. Review of M. Sanz Morales et al., La (inter)textualidad en Plutarco. Actas del XII Simposio Internacional de la Sociedad Española de Plutarquistas (Cáceres/Coimbra, 2017), Exemplaria Classica 22, 315-319. 
2019:

184. “Los comienzos de la identidad colectiva helénica”, Hélade 5.1 (Anejo Etnicidade e formaçâo das identidades no mundo de Homero), 37-55.

185. "Connective particles and literary units in Attic forensic speeches", in: Georgios K. Giannakis et al. (eds), Studies in Greek Lexicography, Berlin/Boston, 219-232.

186. “El papiro de Derveni en su contexto dialectal”, in: Juan Piquero/Pablo de Paz/Soraya Planchas (eds), Nunc est Bacchandum. Homenaje a Alberto Bernabé, Madrid, 359-368.

187. Review of I. Rodríguez Alfageme, Gramática Griega (Madrid, 2018), Estudios Clásicos 155-156, 187-189.

188. "Dialectally hybrid inquiries in the Dodona lamellae", in collaboration with Georgios K. Giannakis, Linguarum Varietas 8, 43-61.

\section{Forthcoming:}

189. "The Structure of the Noun Phrase", in: Antonin Bartonek/Christos Tzitzilis (eds), Ancient Greek Dialects, Thessaloniki.

190. "Graphic Variations in Four Manuscripts by Nemesion, Son of Zoilos", in: Klaas Bentein/Mark Janse (eds), Varieties of Post-classical and Byzantine Greek: novel questions \& approaches, Berlin/New York.

191. “The Origin of to as an Appellative Discourse Marker”, Festchrift in honor of Michael Meier-Brüger.

192. “Linguistic Variation in Macedonian”, in: Rudolf Wachter (ed.), Acts of the Seventh International Colloquium in Ancient Greek Dialects.

193. “Clases semánticas de adverbios de foco en griego clásico”, in: Jesús de la Villa (ed.), Forum classicorum, Madrid.

194. "Sintaxis griega: concepto, objetivos, métodos de análisis", in: Mạ Dolores Jiménez López (ed.), Sintaxis del griego antiguo, Madrid, Anejos de Emerita. 
